\title{
Poor sleep is associated with higher blood pressure in pregnancy - a prospective cohort study
}

\author{
Yafang Tang ${ }^{1}$, Jun Zhang ${ }^{2}$, Fei Dai ${ }^{1}$, Nurul Razali ${ }^{3}$, Shephali Tagore ${ }^{1}$, Bernard, Su Min \\ Chern $^{1}$, and Kok Hian Tan ${ }^{4}$ \\ ${ }^{1}$ KK Women's and Children's Hospital \\ ${ }^{2}$ Xinhua Hospital, School of Medicine, Shanghai Jiao Tong University \\ ${ }^{3} \mathrm{KK}$ Womens and Childrens Hospital, Singapore \\ ${ }^{4} \mathrm{KK}$ Womens and Childrens Hospital
}

July 21,2020

\begin{abstract}
Objective: To elucidate the correlation between sleep disturbances and blood pressure during pregnancy in women with no pre-existing hypertension. Design: Prospective cohort study. Setting: Outpatient specialist clinics at KK Women's and Children's Hospital, Singapore. Population: Women with viable singleton pregnancies confirmed by ultrasonography at less than 14 weeks of amenorrhea at first visit. Methods: 926 subjects were recruited for this study in the outpatient specialist clinics at KK Women's and Children's Hospital, Singapore, between September 1, 2010, and August 31, 2014. They were followed up throughout pregnancy with sleep quality, blood pressure and uterine artery doppler assessed at each visit. Main outcome measures: sleep quality, blood pressure and uterine artery doppler. Results: Sleep progressively worsened as pregnancy advances. Shorter sleep duration and poorer sleep efficiency were associated with higher blood pressure, especially in the first trimester. Mixed model analysis demonstrated overall positive correlation between sleep quality represented by Pittsburgh Sleep Quality Index (PSQI) score and diastolic blood pressure (DBP) $(\mathrm{p}<0.001)$ and mean arterial pressure $(\mathrm{MAP})(\mathrm{p}=0.005)$ during pregnancy after considering all trimesters. Sleep duration was found to be negatively correlated with both systolic blood pressure (SBP) $(\mathrm{p}=0.029)$ and DBP $(\mathrm{p}=0.002)$ while sleep efficiency is negatively correlated with DBP $(\mathrm{p}=0.002)$ only. Overall poor sleep during pregnancy was also found to be correlated to higher uterine artery pulsatility index. Conclusion: Our prospective study demonstrated that sleep quality is significantly correlated with blood pressure during pregnancy with most prominent effect in the first trimester.
\end{abstract}

\section{INTRODUCTION}

The association between sleep disturbances and elevated blood pressure has been well studied in the general population ${ }^{1-4}$. However, relatively few studies focused on the association between sleep quality and blood pressure during pregnancy. Sleep disturbances are very common throughout pregnancy ${ }^{5-6}$. Besides the adverse psychosocial effect of poor sleep, it can also lead to significant adverse maternal and foetal outcomes. Studies have shown that sleep disturbances are associated with pregnancy induced hypertension, gestational diabetes, pre-eclampsia and even adverse cardiac events ${ }^{7-13}$. Blood pressure varies throughout pregnancy. A cohort study by Grindeheim et all showed that both systemic blood pressure (SBP) and diastolic blood pressure (DBP) normally fall in early pregnancy by 5-10 $\mathrm{mm} \mathrm{Hg}$, reaching a mean of $105 / 60 \mathrm{~mm} \mathrm{Hg}$ in the $2^{\text {nd }}$ trimester, and then gradually rise to pre-pregnancy values by term ${ }^{14}$. William et al showed similar pattern ${ }^{15}$. However, one prospective study using 24-hour ambulatory blood pressure monitoring for 4 stages of pregnancy (9-17 weeks, 18-22 weeks, 26-30 weeks and $>30$ weeks of amenorrhea) showed average SBP/DBP of $130 / 77,132 / 79,133 / 81,135 / 86$ respectively, with no dip ${ }^{16}$. While sleep and BP change during pregnancy and relationship of sleep and BP in non-pregnant individuals were well studied, studies investigating the 
effect of sleep on BP during pregnancy are relatively limited, the majority of which focused on the effect of sleep disturbances on the risk of pregnancy induced hypertension. Thus far, there is no cohort study focusing on sleep quality throughout pregnancy along with blood pressure changes among the pregnant population with no pre-existing hypertension.

Uterine artery doppler studies have long been employed to identify patients at risk of developing hypertensive disorders in pregnancy especially preeclampsia. First, second and third trimester uterine artery pulsatilty indexes have been found to be of modest predictive value of developing preeclampsia ${ }^{17-18}$. Abnormal dopplers in early trimesters are associated with subsequent perinatal complications ${ }^{19}$. The effect of sleep on uterine artery doppler was not reported in the literature.

In this prospective study, we aim to delineate the correlation between sleep quality and blood pressure throughout the entire pregnancy in individuals with no pre-existing hypertensive disorder. Uterine artery resistance and pulsatility indexes were also assessed in relation to sleep quality.

\section{MATERIALS AND METHODS}

\section{NORA study}

The Neonatal and Obstetric Risk Assessment (NORA) study is a longitudinal prospective study conducted in KK Women's and Children's Hospital, Singapore, between September 1, 2010, and August 31, 2014. Participants are women with singleton pregnancies who attended antenatal clinics from $1^{\text {st }}$ trimester. Women with viable singleton pregnancies confirmed by ultrasonography at less than 14 weeks of amenorrhea were considered eligible. Patients with chronic medical conditions known to be associated with adverse pregnancy outcomes (such as systemic lupus erythematosus) and pregnancies complicated by aneuploidy or foetal anomalies were excluded. These women were followed up throughout the antenatal course. Women with unsuccessful pregnancies (termination, miscarriage were also excluded. Gestational age was calculated from the estimated due date which was confirmed from the ultrasound scan in the $1^{\text {st }}$ trimester based on crown rump length. A written informed consent was obtained from all participants. NORA study was approved by the SingHealth Centralised Institutional Review Board Ethics Committee, Singapore (CIRB Ref No. 2010/214/D). In the present analysis, 10 women who had pre-existing hypertension were excluded, making the final number of subjects 916 .

\section{Maternal demographics}

Information on sociodemographic and lifestyle characteristics was collected mainly during the $1^{\text {st }}$ antenatal visit: age, parity, race, education level, marital status, occupation, income, BMI, lifestyle characteristics such as drinking, smoking, exercise and caffeine intake. BMI and lifestyle characteristics were also recorded in all subsequent visits. Age and BMI were continuous variables while all the other factors were categorical variables.

\section{Sleep assessment}

Sleep quality and BP were assessed at 4 visits: 9 to 14 weeks of gestation (visit 1), 18 to 22 weeks of gestation (visit 2), 28 to 32 weeks of gestation (visit 3) and 34 to 39 weeks of gestation (visit 4). During each visit, sleep quality was assessed via the Pittsburgh Sleep Quality Index (PSQI) questionnaire. Four main questions were asked in the questionnaire: (1) "During the past month, what time have you usually gone to bed at night"; (2) During the past month, how long (in minutes) has it usually taken you to fall asleep each night"; (3) During the past month, what time have you usually got up in the morning"; (4) During the past month, how many hours of actual sleep did you get at night, which may be different from the number of hours you spent in bed". PSQI score was calculated after combining the scores for each question. Women with poorer sleep quality have higher PSQI scores. Furthermore, specific aspects of sleep quality including sleep latency (question 2), sleep duration (question 4) and sleep efficiency (calculated by actual hours of sleep over total hours from time in bed to time of getting up) were also individually assessed and compared in relation to blood pressure. 


\section{Blood pressure and uterine artery doppler assessment}

Non-invasive systolic and diastolic blood pressure was taken during each visit and MAP was calculated from $\mathrm{SBP}$ and DBP with the formula of MAP $=1 / 3 \mathrm{SBP}+2 / 3$ DBP. Uterine artery flow velocity waveforms with colour-pulsed Doppler was performed during each visit via transabdominal ultrasound. Resistance and pulsatility indexes were recorded.

\section{Statistical analysis}

Statistical analysis of the data was performed using SPSS 22.0 (IBM, Armonk, NY, USA). For maternal demographic and lifestyle characteristics, continuous variables were described as mean \pm standard deviation. Categorical variables were described as the sample number and frequency of each category. Step wise linear regression was performed across the four visits on the following variables: PSQI, sleep latency, sleep duration and sleep efficiency to establish the relationship between sleep quality and blood pressure throughout pregnancy, after considering potential confounding factors. Data from each individual visit was analysed separately. Mixed model analysis was employed to study the overall correlation between sleep and BP as well as sleep and uterine artery resistance and pulsatility index, combining the information from all 4 antenatal visits. Analysis was adjusted for possible confounders.

\section{RESULTS}

As shown in Table 1a, our study subjects consist of a multiracial population with average age of 30 years and different educational levels, occupations and social status. Chinese and Malay women made up most of our study subjects (50.7\% and $27.3 \%$ respectively) with small numbers of Indians and others. Most of the subjects have education levels of high school/junior college and above. Their household income ranges from $<3500$ to more than 8500 Singapore dollars a month, with most women working as white-collar workers. Similar percentage of nulliparous (54.4\%) and multiparous (45.6\%) women were included in this study.

Life style, BMI, blood pressure and sleep quality throughout pregnancy were presented in Table 1b. A small percentage of women continued to drink coffee during pregnancy (21-33.4\%). A smaller percentage of women remained actively drinking (0.8-2.5\%) or smoking (1.8-2.6\%) during pregnancy. Average BMI of the patients increased expectedly throughout pregnancy (from 24.1 at the first visit to 28.1 at the last visit). The average SBP/DBP (MAP) in the 4 visits were 108/66 (80) $\mathrm{mmHg}, 109 / 65$ (79) $\mathrm{mmHg}, 110 / 66$ (81) $\mathrm{mmHg}$ and 112/69 (83) $\mathrm{mmHg}$ respectively, showing an overall upward trend. Sleep quality score, as represented by PSQI, had an average range of 6.3-8. The average of individual aspects of sleep quality including sleep duration (6.5-7.0 hours), latency (20-26mins) and efficiency (82-85\%) were also shown in Table 2. Overall sleep quality, sleep efficiency and latency worsen while sleep duration shortens as pregnancy progresses.

During the first visit, it was found that SBP $(\mathrm{p}=0.019)$, DBP $(\mathrm{p}=0.023)$ and MAP $(\mathrm{p}=0.014)$ were all significantly lower in women with longer duration of sleep (Table 2a). Likewise, SBP $(p=0.016)$, DBP $(p=0.017)$ and MAP ( $\mathrm{p}=0.014$ ) were significantly lower in women with better efficiency of sleep (Table 2a). No significant difference in BP was found in women with different sleep qualities during the $2^{\text {nd }}$ and $4^{\text {th }}$ visits (Table 2a). DBP $(\mathrm{p}=0.011)$ and MAP $(\mathrm{p}=0.027)$ were significantly lower in subjects with better sleep efficiency during the $3^{\text {rd }}$ visit (Table 2$)$.

When overall sleep and BP were assessed throughout the whole pregnancy, Lower PSQI score $(\mathrm{p}<0.001)$, shorter sleep latency $(\mathrm{p}=0.008)$ and better sleep efficiency $((\mathrm{p}=0.008)$ were found to be correlated to lower DBP (Table 2b). Longer sleep duration was associated with lower SBP $(\mathrm{p}=0.049)$ and DBP $(\mathrm{p}=0.008)$ (Table $2 \mathrm{~b})$.

Assessment of the overall relationship between sleep and uterine artery doppler throughout pregnancy showed that poorer sleep quality (higher PSQI, longer sleep latency, shorter sleep duration and worse sleep efficiency) were associated with higher UA PI (Table 3). Patients with higher PSQI and shorter sleep duration were also found to have higher UA RI (Table 3).

\section{Discussion}


Our result showed that blood pressure (SBP and/or DBP and/or MAP) was lower in the pregnant women with better sleep quality: shorter sleep latency, longer duration of sleep, better efficiency and lower PSQI scores. UA PI was similarly lower in women with better sleep quality. The correlation between sleep and BP was the most significant in the first trimester as demonstrated by liner regression analysis.

Sleep latency, duration and efficiency were important aspects of sleep with significant correlation to BP. A previous cohort study by William et al. found that mean $1^{\text {st }}$ and $2^{\text {nd }}$ trimester SBP and DBP values were similar among women reporting to be short sleepers ([?] 6 hours) versus women reporting to sleep $>9$ hours. However, both short and long sleep duration in early pregnancy were associated with increased mean $3^{\text {rd }}$ trimester SBP and DBP compared to women who sleep for 9 hours in their study ${ }^{15}$. Their study findings were in line with some previous studies done in the non-pregnant population ${ }^{1-4}$. The analysis in our study differed from these previous studies. Sleep duration, sleep efficiency, latency and PSQI were considered as continuous variables in mixed model and linear regression analysis instead of categorical variables as in those studies. We believe that correlation analysis using continuous variables offers a more comprehensive comparison. The sleep duration for our study subjects fell between 4.7 and 8.7 hours. There was no subject with $>9$ hours of sleep. This may also explain the different findings as William et al's study showed higher $\mathrm{BP}$ in women who slept $>9$ hours.

The mechanism linking sleep and blood pressure is very complex. One possible mechanism is increased oxidative stress and reduced nitric oxide (NO) in poor sleepers. NO is an important mediator in vascular function causing dilatation of the vessels and thus resulting in lower BP. In the case of obstructive sleep apnoea, nocturnal hypoxemia induces oxidative stress which triggers an inflammatory response and reduction in $\mathrm{NO}^{20-21}$. Another possible mechanism is through the sympathetic pathway and the hypothalamic-pituitary-adrenal axis. In some experimental studies, increased secretion of catecholamines, altered sympathovagal balance and disruption in cortisol levels were postulated to link poor sleep with elevated blood pressure ${ }^{22-25}$. A few interesting studies showed that increase in pro-inflammatory cytokines such as Interleukin- 1 and Tumour necrosis factor-alpha alter sleep pattern and may potentially cause elevated blood pressure ${ }^{26-28}$. However, the exact underlying mechanism remains to be elucidated.

UA PI and RI were significantly lower in women with better sleep as shown in our study. The association between sleep quality and uterine artery blood flow was reported in literature. One postulation is that poor sleep may lead to suboptimal trophoblastic invasion of myometrium and consequently higher blood flow resistance. In other words, higher blood pressure in people with poorer sleep can potentially be contributed by placental dysfunction. Another possible explanation is that sleep is associated with worse uterine artery doppler and higher blood pressure via a common mechanism such as reduced NO which leads to vasoconstriction.

\section{Strength and Limitations of study}

Our study is the first large cohort study that demonstrated gradual change in sleep quality along with blood pressure changes throughout pregnancy, via which the correlation between sleep and BP was better scrutinized. As sleep pattern may gradually change over time, sleep quality in the first visit may not be representative of the overall sleep pattern throughout pregnancy. The correlation between sleep and BP were individually assessed at each visit and that accounts for the most direct correlation if there is any. Follow up rate of our study is very high which makes the data more reliable.

Blood pressure varies throughout the day and is affected by activities immediately prior to blood pressure measurement. Our study only used a single point blood pressure during each visit for assessment. This may cause some inaccuracy and error as it may not be representative of the actual blood pressure. If possible, 24-hour ambulatory pressure would be a better modality for the accurate assessment of blood pressure, although it is logistically much more difficult to execute. Presence of sleep disorders such as sleep apnoea and subjects' psychiatric history such as and history of depression were not explored in our study. These factors can potentially affect sleep quality and blood pressure and it would be ideal if these factors can be adjusted in the analysis. 


\section{Implications of study}

The correlation between sleep disturbances and higher blood pressure urges clinicians and researchers to recognize the importance of sleep during pregnancy and explore ways to improve sleep antenatally. Screening for sleep disruption is not routinely done in most obstetric units. Short questionnaire on sleep quality can be incorporated into routine obstetric care. Early identification of patients at risk of sleep disturbances and intervention at early stage may offer some benefit in pregnancy outcome. Studies on potential interventions in improving sleep in pregnancy are rather limited. Stremler $\mathrm{R}$ et al conducted a randomized controlled trial on the effect of behavioural-educational intervention in improving sleep in postpartum women. Although no significance was found in the primary outcome of nocturnal sleep, it is still worth exploring intervention during early pregnancy instead of postpartum when sleep is interfered by breastfeeding significantly. Another interesting study recently reported significant improvement of sleep in terms of longer sleep duration and less sleep disruption with home-based cognitive-behavioural training program during late pregnancy ${ }^{29}$. Further studies on possible interventions antenatally to improve sleep and potentially lower blood pressure would be valuable.

\section{Conclusion}

Our cohort study is among the first to show the correlation between sleep and blood pressure throughout pregnancy in the population without pre-existing hypertensive disorder. Most significant correlation was found in the first trimester. Uterine artery doppler was worse in poorer sleepers suggesting a potential association between sleep and placental function which may affect blood pressure. Future studies should focus on interventions to improve sleep antenatally with an effort to optimize blood pressure and pregnancy outcome.

\section{Acknowledgement}

The authors thank all the members involved in Integrated Platform for Research in Advancing Metabolic Health Outcomes in Women and Children (IPRAMHO) and NORA study for their invaluable contribution.

\section{Disclosure of interests}

The authors have no conflict of interest to declare

\section{Contribution to authorship}

Y.T. contributed to the design of study, data analysis and interpretation, manuscript drafting and review. J.Z., F.D., KH.T. contributed to design of study, data analysis, data interpretation and review of manuscript to be published. KH.T., NS.R., S.T., B.C. contributed to conception, design of study and review of manuscript to be published.

\section{Details of ethical approval}

A written informed consent was obtained from all participants and the study was approved by the SingHealth Centralised Institutional Review Board Ethics Committee, Singapore (CIRB Ref No. 2010/214/D).

\section{Funding}

This study is supported by Singapore National Medical Research Council (NMRC) Collaborative Centre Grant grant-Integrated Platform for Research in Advancing Metabolic Health Outcomes of Women and Children, IPRAMHO/CGAug16C008) and Neonatal and Obstetrics Risks Assessment (NORA) prospective cohort study (Project Grant number NMRC/PPG/KKH/2010)

\section{REFERENCES}

1. Cappuccio FP, Stranges S, Kandala NB, et al., Gender-specific associations of short sleep duration with prevalent and incident hypertension: the Whitehall II Study. Hypertension.2007;50:693-700. 
2. Gangwisch JE, Heymsfield SB, Boden-Albala B, et al., Short sleep duration as a risk factor for hypertension: analyses of the first National Health and Nutrition Examination Survey. Hypertension $2006 ; 47: 833-9$

3. Kim J, Jo I, authors. Age-dependent association between sleep duration and hypertension in the adult Korean population. Am J Hypertens2010; 23:1286-91.

4. Knutson KL, Van CE, Rathouz PJ, et al., authors. Association between sleep and blood pressure in midlife: the CARDIA sleep study.Arch Intern Med 2009; 169:1055-61.

5. Mindell JA, Jacobson BJ. Sleep disturbances during pregnancy. J Obstet Gynecol Neonatal Nurs 2000; 29:590-7.

6. Pien GW, Schwab RJ. Sleep disorders during pregnancy. Sleep2004; 27:1405-17.

7. Zafarghandi N, Hadavand S, Davati A, Mohseni SM, Kimiaiimoghadam F, Torkestani F. The effects of sleep quality and duration in late pregnancy on labor and fetal outcome. J Matern Fetal Neonatal Med 2012; 25:535-7.

8. Reutrakul S, Zaidi N, Wroblewski K, Kay HH, Ismail M, Ehrmann DA, Van Cauter E. Sleep disturbances and their relationship to glucose tolerance in pregnancy. Diabetes Care 2011; 34:2454-7

9. Okun ML, Schetter CD, Glynn LM. Poor sleep quality is associated with preterm birth. Sleep 2011; 34:1493-8.

10. Ding XX, Wu YL, Xu SJ, Zhang SF, Jia XM, Zhu RP, Hao JH, Tao FB. A systematic review and quantitative assessment of sleep-disordered breathing during pregnancy and perinatal outcomes. Sleep Breath 2014;18:703-13

11. Kajeepeta S1, Sanchez SE, Gelaye B, Qiu C, Barrios YV, Enquobahrie DA, Williams MA. Sleep duration, vital exhaustion, and odds of spontaneous preterm birth: a case-control study. BMC Pregnancy Childbirth2014; 14:337.

12. Carnelio S, Morton A, McIntyre HD., Sleep disordered breathing in pregnancy: the maternal and fetal implications. J Obstet Gynaecol 2017; 37:170-178.

13. Chang, J. J., Pien, G. W., Duntley, S. P., \& Macones, G. A. Sleep deprivation during pregnancy and maternal and fetal outcomes: Is there a relationship? Sleep Medicine Reviews 2010; 14: 107-114

14. Grindheim G, Estensen ME, Langesaeter E, Rosseland LA, Toska K, authors. Changes in blood pressure during healthy pregnancy: a longitudinal cohort study. J Hypertens 2012;30:342-50

15. Williams MA, Miller RS, Qiu C, Cripe SM, Gelaye B, Enquobahrie D. Associations of early pregnancy sleep duration with trimester-specific blood pressures and hypertensive disorders in pregnancy. Sleep 2010; 33: 1363-71

16. Brown MA, Robinson A, Bowyer L, Buddle ML, Martin A, Hargood JL, Cario GM. Ambulatory blood pressure monitoring in pregnancy: what is normal? Am J Obstet Gynecol 1997; 178:836-842.

17. Cnossen JS, Morris RK, ter Riet G, Mol BW, van der Post JA, Coomarasamy A, Zwinderman AH, Robson SC, Bindels PJ, Kleijnen J, Khan KS. Evaluation of 7 Serum Biomarkers and Uterine Artery Doppler Ultrasound for First-Trimester Prediction of Preeclampsia: A Systematic Review. CMAJ. 2008;178:701-11.

18. Običan SG, Odibo L, Tuuli MG, Rodriguez A, Odibo AO.Third trimester uterine artery Doppler indices as predictors of preeclampsia and neonatal small for gestational age.J Matern Fetal Neonatal Med. 2019; 13:1-6.

19. Giordano R, Cacciatore A, Romano M et al., Uterine artery doppler flow studies in obstetric practice. J Prenat Med 2010; 4: 59-62.

20. Haney A, Buysse DJ, Okun M. Sleep and pregnancy-induced hypertension: a possible target for intervention? J Clin Sleep Med 2013; 9:1349-56.

21. Peker Y, Hedner J, Norum J, Kraiczi H, Carlson J, authors. Increased incidence of cardiovascular disease in middle-aged men with obstructive sleep apnea: a 7-year follow-up. Am J Respir Crit Care Med 2002;166:159-65.

22. Spiegel K, Leproult R, Van Cauter E, authors. Impact of sleep debt on metabolic and endocrine function. Lancet 1999; 354:1435-9.

23. Lusardi P, Zoppi A, Preti P, Pesce RM, Piazza E, Fogari R. Effects of insufficient sleep on blood 
pressure in hypertensive patients: a 24-h study. Am J Hypertens 1999; 12:63-8.

24. Kato M, Phillips BG, Sigurdsson G, Narkiewicz K, Pesek CA, Somers VK. Effects of sleep deprivation on neural circulatory control.Hypertension 2000; 35:1173-5.

25. Spath-Schwalbe E, Gofferje M, Kern W, Born J, Fehm HL. Sleep disruption alters nocturnal ACTH and cortisol secretory patterns.Biol Psychiatry 1991; 29:575-84.

26. Obal F Jr, Opp M, Cady AB, et al. Interleukin 1 alpha and an interleukin 1 beta fragment are somnogenic. Am J Physiol 1990; 259:R439-46.

27. Kapas L, Hong L, Cady AB, et al. Somnogenic, pyrogenic, and anorectic activities of tumor necrosis factor-alpha and TNF-alpha fragments. Am J Physiol 1992; 263:R708-15.

28. Williams MA, Farrand A, Mittendorf R, et al. Maternal second trimester serum tumor necrosis factoralpha-soluble receptor p55 (sTNFp55) and subsequent risk of preeclampsia. Am J Epidemiol 1999; 149:323-9.

29. Lee KA, Gay CL, Alsten CR. Sleep Enhancement Training for Pregnant Women. Obstet Gynecol 2016; 128(5):964-971.

\section{Hosted file}

BJOG_Tables.docx available at https://authorea.com/users/344873/articles/471230-poor-sleepis-associated-with-higher-blood-pressure-in-pregnancy-a-prospective-cohort-study 